\title{
Comments on "Coexistence of hidden chaotic attractors in a novel no-equilibrium system" (Nonlinear Dyn, doi:10.1007/s11071-016-3170-x)
}

\author{
Ivo Petráš $\mathbb{D}$
}

Received: 25 February 2017 / Accepted: 10 July 2017 / Published online: 18 July 2017

(C) Springer Science+Business Media B.V. 2017

\begin{abstract}
In this comment, an enhancement of issue published in the paper "Coexistence of hidden chaotic attractors in a novel no-equilibrium system" (Nonlinear Dyn, doi:10.1007/s11071-016-3170-x) is addressed. We have shown that the proposed novel autonomous chaotic system can be extended to its fractional-order version where hidden attractors as well as other dynamical properties of the new no-equilibrium system can be observed. A created MATLAB function for the new fractional-order no-equilibrium system is also presented.
\end{abstract}

Keywords Fractional calculus - Fractional-order chaotic system · Grünwald-Letnikov derivative

\section{Introduction and motivation}

Investigation of chaotic systems has a long tradition. Basically, there are two kinds of attractors observed in chaotic systems: self-excited attractor and hidden attractor. It is well-known that hidden attractors cannot be localized by using the standard computational procedure and therefore it is difficult to predict the existence of them in system. However, hidden attractors appear in numerous dynamical systems [6,9-11]. Moreover, there is a significant interest in studying hidden attractors because they play an important role in both theo-

\section{Petráš $(\varangle)$}

Faculty of BERG, Technical University of Košice,

B. Němcovej 3, Košice 042 00, Slovak Republic

e-mail: ivo.petras@tuke.sk retical problems and practical engineering applications [10]. For more details see also survey paper [5].

On the other hand, the subject of fractional calculus has gained considerable popularity and importance mainly during the past three decades $[8,13]$.

Fractional (order) calculus, also non-integer order calculus, is known since the classical calculus with the first written reference dated September 1695 in the correspondence between Leibniz and L'Hospital. Nowadays, the fractional calculus has a wide area of applications in various fields $[4,8,13]$ as well as in the chaotic systems theory and applications [12].

Based on above consideration, it is possible to combine the fractional calculus into description of the novel chaotic systems where the hidden attractors appeared.

This comment is organized as follows. In Sect. 1, the introduction to the problem and motivation is briefly discussed. Section 2 is focused on fractional calculus fundamentals. Section 3 brings a new fractionalorder chaotic system. In Sect. 4, the simulation results and discussion are described. Section 5 concludes this note with some additional remarks for further research investigation.

\section{Preliminaries}

\subsection{Definition of fractional-order operator}

Fractional calculus is a generalization of integration and differentiation to non-integer (fractional) order fundamental operator ${ }_{a} D_{b}^{\alpha}$, where $a$ and $b$ are 
the bounds of the operation. The standard notation for denoting the left-sided fractional-order integrodifferential operator of a function $f(t)$ defined in the interval $[a, b]$ is ${ }_{a} D_{t}^{\alpha} f(t)$, with $\alpha \in \mathrm{R}$.

There exist many definitions for fractional operator (fractional order integrals and derivatives), but in this article we will focus only on the Grünwald-Letnikov definition (GLD), which is given as follows [8,13]:

${ }_{a} D_{t}^{\alpha} f(t)=\lim _{h \rightarrow 0} \frac{1}{h^{\alpha}} \sum_{j=0}^{\left\lfloor\frac{t-a}{h}\right\rfloor}(-1)^{j}\left(\begin{array}{c}\alpha \\ j\end{array}\right) f(t-j h)$,

where $\lfloor z\rfloor$ is the floor function, i.e., the greatest integer smaller than $z$, and

$\left(\begin{array}{c}\alpha \\ j\end{array}\right)=\frac{\alpha !}{j !(\alpha-j) !}=\frac{\Gamma(\alpha+1)}{\Gamma(j+1) \Gamma(\alpha-j+1)}$

are the binomial coefficients for $\left(\begin{array}{l}\alpha \\ 0\end{array}\right)=1$. This form of definition is very helpful for obtaining numerical solutions of fractional differential equations. However, we must be careful with the initial conditions setting because it could be a complex problem in some systems.

\subsection{Numerical solution of fractional differential equations}

For numerical calculation of fractional-order derivatives, we can use the relation (3) derived from the GLD (1). This approach is based on the fact that for a wide class of functions, the most of definitions are equivalent. The relation for the explicit numerical approximation of $q$-th derivative at the points $k h,(k=1,2, \ldots)$ has the following form [13]:

${ }_{\left(k-L_{\mathrm{m}} / h\right)} D_{t_{k}}^{q} f(t) \approx h^{-q} \sum_{j=0}^{k} c_{j}^{(q)} f\left(t_{k-j}\right)$,

where $L_{\mathrm{m}}$ is the "memory length", $t_{k}=k h, h$ is the time step of calculation (definition (3) is valid only as $h$ tends toward 0 and that the accuracy of the simulation depends on the value of $h)$ and $c_{j}^{(q)}(j=0,1, \ldots)$ are binomial coefficients. For their calculation we can use the following expression:

$c_{0}^{(q)}=1, \quad c_{j}^{(q)}=\left(1-\frac{1+q}{j}\right) c_{j-1}^{(q)}$.

Then, general numerical solution of the fractional differential equation

${ }_{0} D_{t}^{q} y(t)=f(y(t), t)$, can be expressed as

$y\left(t_{k}\right)=f\left(y\left(t_{k}\right), t_{k}\right) h^{q}-\sum_{j=0}^{k} c_{j}^{(q)} y\left(t_{k-j}\right)$.

For the memory term expressed by the sum, a "short memory" principle can be used for various $L_{\mathrm{m}}$.

An evaluation of the short memory effect and convergence relation of the error between short and long memory were clearly described and also proved in [13].

\section{Model of the new fractional-order system}

In paper [11], the simple case of the new chaotic system without equilibrium was described in the following form:

$\dot{x}(t)=y(t)$,

$\dot{y}(t)=-x(t)-y(t) z(t)$,

$\dot{z}(t)=|x(t)|+x(t) y(t)-a$,

in which $a$ is the positive parameter $(a>0)$. The dynamical properties of the new system (6) as for instance equilibrium points (there is no equilibrium in (6)), dissipativity, chaotic behavior for various values of parameter $a$ as for example Poincaré map, bifurcation diagram, Lyapunov exponents, and chaotic attractors have been investigated in paper [11]. Moreover, an electronic circuit implementation of the system (6), which included 15 resistors $(10 \mathrm{k} \Omega), 3$ capacitors $(10 \mathrm{nF}), 7$ operational amplifiers, 2 diodes, 2 analog multipliers and the power supplies $\left( \pm 15 \mathrm{~V}_{\mathrm{DC}}\right)$ was built. In the circuit are three integrators, which are created by the operational amplifiers together with the capacitors in feed-back.

Generally, real electrical elements as resistor, capacitor, coil and memristor are nonlinear and fractional order; however, their models are very often simplified [12]. This notion is based on fact that there is not ideal electrical element. Now, we already know that all real elements lie in between two. For instance, resistor created by wire has property of resistor and coil too. Moreover, we know element fractor (between resistor and capacitor) as well as fractductor (between resistor and coil).

Westerlund and Ekstam [14] in 1994 proposed a new linear capacitor model. It is based on Curie's empirical law of 1889 which states that the current through a capacitor is 
$I(t)=\frac{V_{0}}{h_{1} t^{\alpha}}$,

where $h_{1}$ and $\alpha$ are constant, $V_{0}$ is the DC voltage applied at $t=0$, and $0<\alpha<1,(\alpha \in \mathrm{R})$.

For a general input voltage $V(t)$, the current is

$I(t)=C \frac{\mathrm{d}^{\alpha} V(t)}{\mathrm{d} t^{\alpha}} \equiv C_{0} D_{t}^{\alpha} V(t)$,

where $C$ is the capacitance of the capacitor. It is related to the kind of dielectric. Another constant $\alpha$ (order) is related to the losses of the capacitor. Westerlund and Ekstam provided in their work the table of various capacitor dielectrics with appropriate constant $\alpha$ which has been obtained experimentally by measurements.

For a current in the capacitor the voltage is

$V(t)=\frac{1}{C} \int_{0}^{t} I(t) \mathrm{d} t^{\alpha} \equiv \frac{1}{C}{ }_{0} D_{t}^{-\alpha} I(t)$.

Taking into account above consideration on capacitor, the new mathematical model of the real circuit implementation of the chaotic system (6) can be derived. Similarly to method provided in book [12], we can apply Kirchhoff's circuit laws into the circuit depicted in Fig. 9 in paper [11] and derive a new fractional-order model of the chaotic system (6) in the following dimensionless form:

${ }_{0} D_{t}^{q_{1}} x(t)=y(t)$,

${ }_{0} D_{t}^{q_{2}} y(t)=-x(t)-y(t) z(t)$,

${ }_{0} D_{t}^{q_{3}} z(t)=|x(t)|+x(t) y(t)-a$,

where $q_{1}, q_{2}, q_{3}$ are the real orders of used capacitors.

For simulation purposes, we will use a numerical solution of equations (9) obtained by using the relationship (3), derived from the GLD (1), which leads to equations in the form:

$$
\begin{aligned}
x\left(t_{k}\right)= & {\left[y\left(t_{k-1}\right)\right] h^{q_{1}}-\sum_{j=0}^{k} c_{j}^{\left(q_{1}\right)} x\left(t_{k-j}\right), } \\
y\left(t_{k}\right)= & {\left[-x\left(t_{k-1}\right)-y\left(t_{k-1}\right) z\left(t_{k-1}\right)\right] h^{q_{2}} } \\
& -\sum_{j=0}^{k} c_{j}^{\left(q_{2}\right)} y\left(t_{k-j}\right), \\
z\left(t_{k}\right)= & {\left[\left|x\left(t_{k-1}\right)\right|+x\left(t_{k-1}\right) y\left(t_{k-1}\right)-a\right] h^{q_{3}} } \\
& -\sum_{j=0}^{k} c_{j}^{\left(q_{3}\right)} z\left(t_{k-j}\right),
\end{aligned}
$$

where $T_{\text {sim }}$ is the simulation time, $k=1,2,3 \ldots, N$, for $N=\left[T_{\operatorname{sim}} / h\right]$, and $(x(0), y(0), z(0))$ is the start

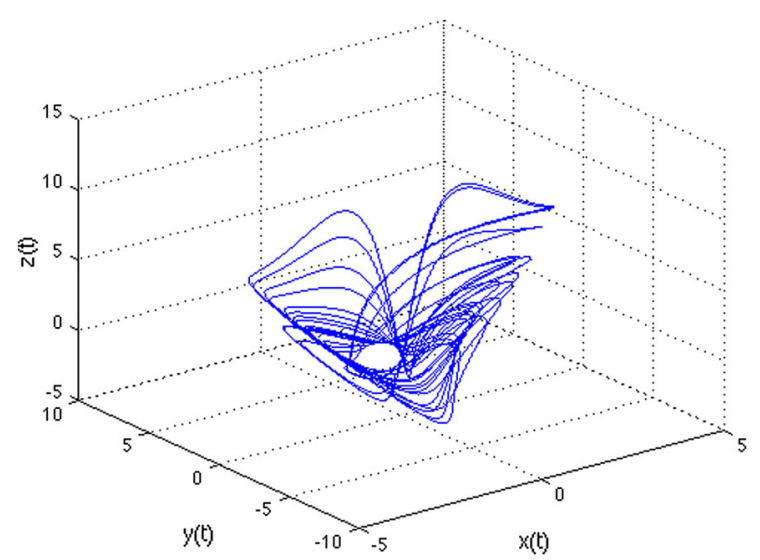

Fig. $1 x-y-z$ state space of system (6) for $a=1.35$

point (initial conditions). The binomial coefficients $c_{j}^{\left(q_{i}\right)}, \forall i$, are calculated according to relation (4). Proposed numerical solution (10) was implemented as the MATLAB function, which syntax is listed in Appendix.

\section{Simulation results and comments}

First, let us use proposed numerical solution (10) to simulate chaotic system (6) for the parameter $a=1.35$, simulation time $T_{\text {sim }}=300 \mathrm{~s}$, time step $h=0.005$, and initial conditions $(x(0)=0, y(0)=0.1, z(0)=0)$. Using created MATLAB function, we can call it as follows:

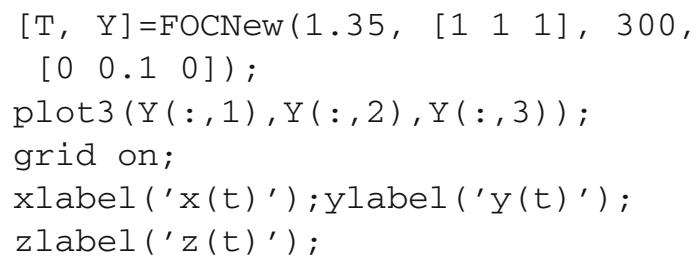

In Fig. 1 is depicted chaotic behavior of the system (6) for $a=1.35$ and the initial conditions $(x(0), y(0), z(0))=(0,0.1,0)$ in state space.

Due to the fact that parameter $a$ should be within intervals $1.305<a<1.381$ or $1.224<a<1.303$ in order to observe chaotic behavior of the system, let us move from first interval to the second one and consider $a=1.3$. Using created MATLAB function, we can call it as follows:

\section{$[\mathrm{T}, \mathrm{Y}]=\mathrm{FOCNew}\left(1.3,\left[\begin{array}{lll}1 & 1 & 1\end{array}\right], 300\right.$, $\left.\left[\begin{array}{lll}0 & 0.1 & 0\end{array}\right]\right)$;}

In Fig. 2 is depicted chaotic behavior of the system (6) for $a=1.3$ and the initial conditions $(x(0), y(0), z(0))=(0,0.1,0)$ in state space. 


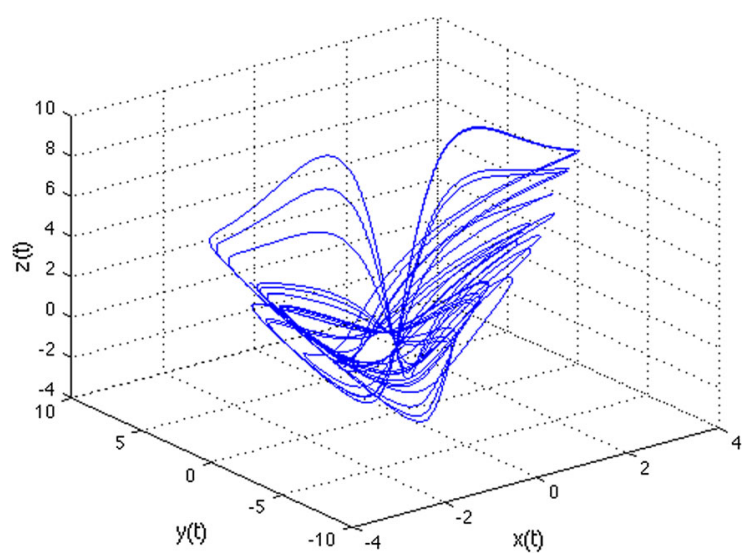

Fig. $2 x-y-z$ state space of system (6) for $a=1.3$

Now, let us consider the fractional-order system (9), where we assume real order of the identical capacitors $q_{1}=q_{2}=q_{3}=0.98$, for the parameter $a=1.3$, simulation time $T_{\text {sim }}=300 \mathrm{~s}$, time step $h=0.005$, and initial conditions $(x(0)=0, y(0)=0.1, z(0)=0)$. Using created MATLAB function, we can call it as follows:

$\mathrm{q}=0.98$;

$[\mathrm{T}, \mathrm{Y}]=\mathrm{FOCNew}(1.3,[\mathrm{q}$ q q $], 300$,

$\left.\left[\begin{array}{lll}0 & 0.1 & 0\end{array}\right]\right)$;

In Figs. 4-6 are depicted chaotic behavior of the system (9) for $a=1.3$, real orders $q_{1}=q_{2}=q_{3}=$ 0.98 , and the initial conditions $(x(0), y(0), z(0))=$ $(0,0.1,0)$ in state planes (blue color), respectively. We can observe chaotic behavior when the total system order is 2.94 .

In order to have more details, in Fig. 3 is presented the bifurcation diagram of the variable $x$ versus the parameter $q$ within interval $[0.93 ; 1]$.

The Lyapunov exponents of system (9) for $a=1.3$ and orders $\bar{q}=0.98$, computed by method described in [15], are: 0.1947, 0, -4.5101, which confirm chaos.

In addition, let us investigate existence of the coexisting attractors. We will consider the same orders and parameter as in previous example of the fractionalorder system (9), but for different initial conditions $(x(0)=0, y(0)=-0.1, z(0)=0)$. Using created MATLAB function, we can call it as follows:

$q=0.98$;

$[\mathrm{T}, \mathrm{Y}]=\mathrm{FOCNew}(1.3,[\mathrm{q}$ q q $], 300$,

$\left.\left[\begin{array}{lll}0 & -0.1 & 0\end{array}\right]\right)$;

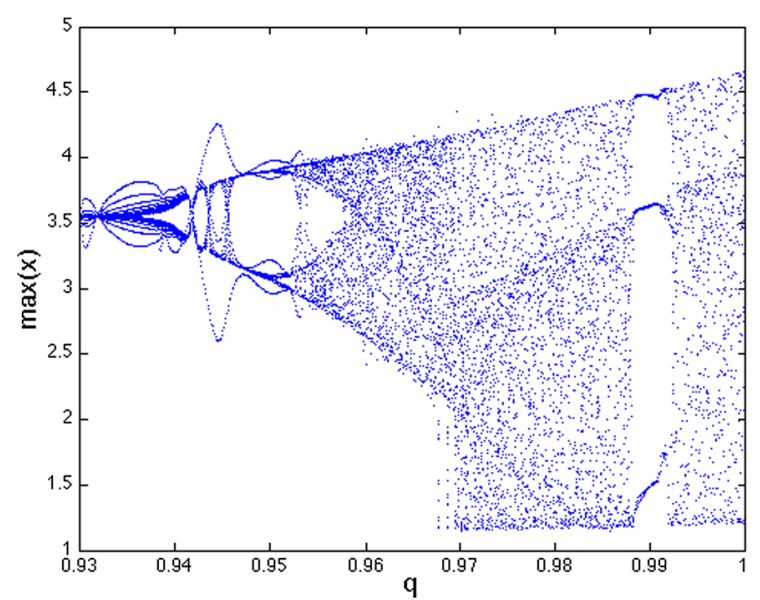

Fig. 3 Bifurcation diagram of system (9) when changing the bifurcation parameter $q$ for the selected initial conditions $(x(0), y(0), z(0))=(0,0.1,0)$ and fixed parameter $a=1.3$

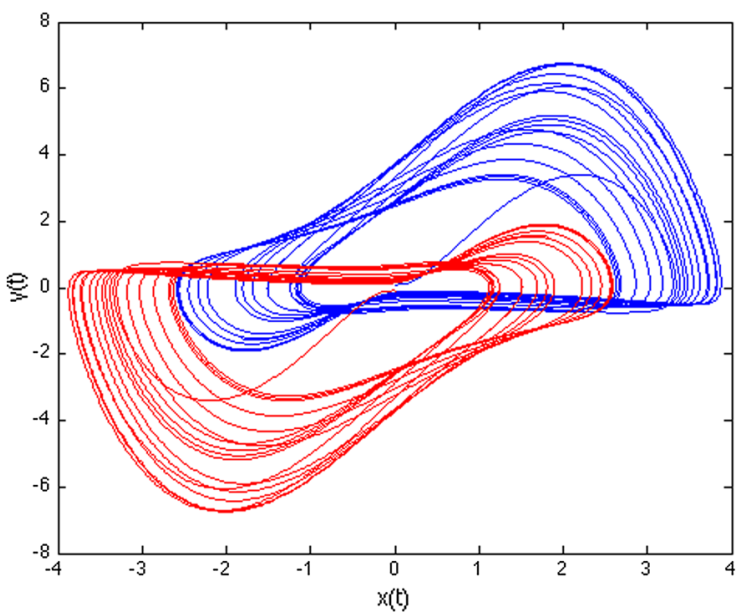

Fig. $4 x-y$ state plane of system (9) for $a=1.3$

In Figs. 4, 5 and 6 are depicted chaotic behavior, coexisting attractors, of the system (9) for $a=1.3$, real orders $q_{1}=q_{2}=q_{3}=0.98$, and the initial conditions $(x(0), y(0), z(0))=(0,0.1,0)$ (blue color) and the initial conditions $(x(0), y(0), z(0))=(0,-0.1,0)$ (red) in state planes (red color), respectively.

\section{Conclusions}

In this comment paper, we pointed out that the results presented in paper [11] can be enhanced. In spite of the fact that theory of fractional-order nonlinear (chaotic) systems is known and it was described in many papers 


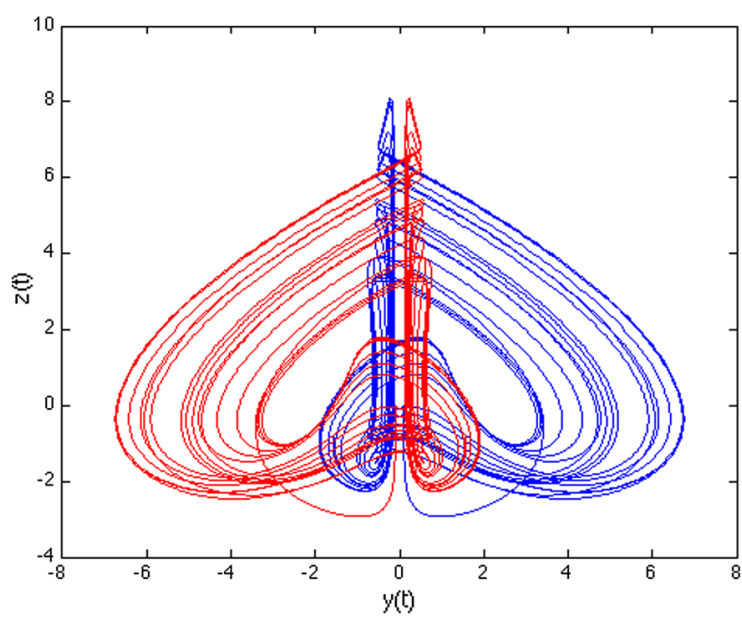

Fig. $5 y-z$ state plane of system (9) for $a=1.3$

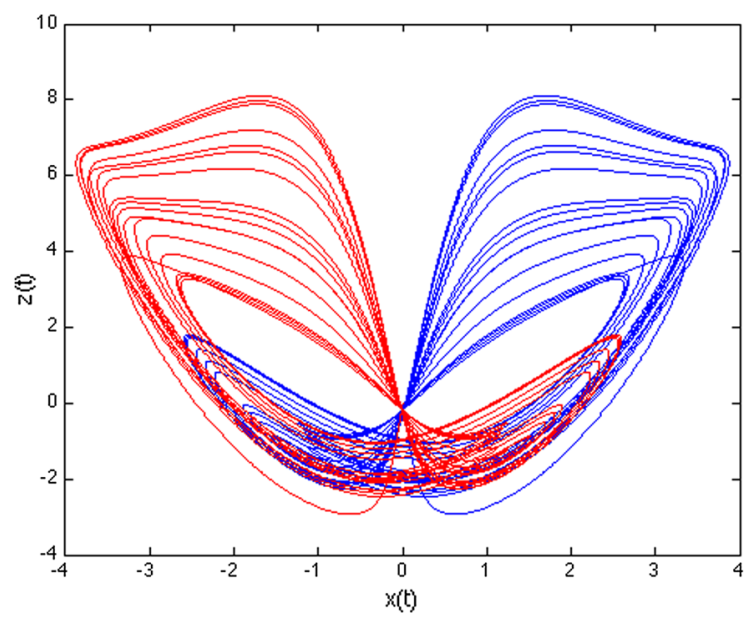

Fig. $6 x-z$ state plane of system (9) for $a=1.3$

and books $[4,7,12]$, it was just for the system with known equilibrium, except few of them, see, e.g., [1-3].

In this note, a contribution to new phenomenon and almost unexplored area of a fractional-order chaotic no-equilibrium system was described together with the tool (MATLAB function) for its further investigation, even the coexisting attractors. Such nonlinear system without equilibrium is appropriate for practical applications. We have more degree of freedom due to the orders.

Acknowledgements This work was supported in part by the Slovak Grant Agency for Science under grant VEGA 1/0908/15, and by the Slovak Research and Development Agency under the contracts No. APVV-14-0892 and No. SK-PL-2015-0038, US project ARO W911NF-15-1-0228, and COST Action CA15225 a network supported by COST (European Cooperation in Science and Technology).

\section{Appendix}

Implementation of the proposed numerical solution (10) as the MATLAB function FOCNew ( ). See its syntax below.

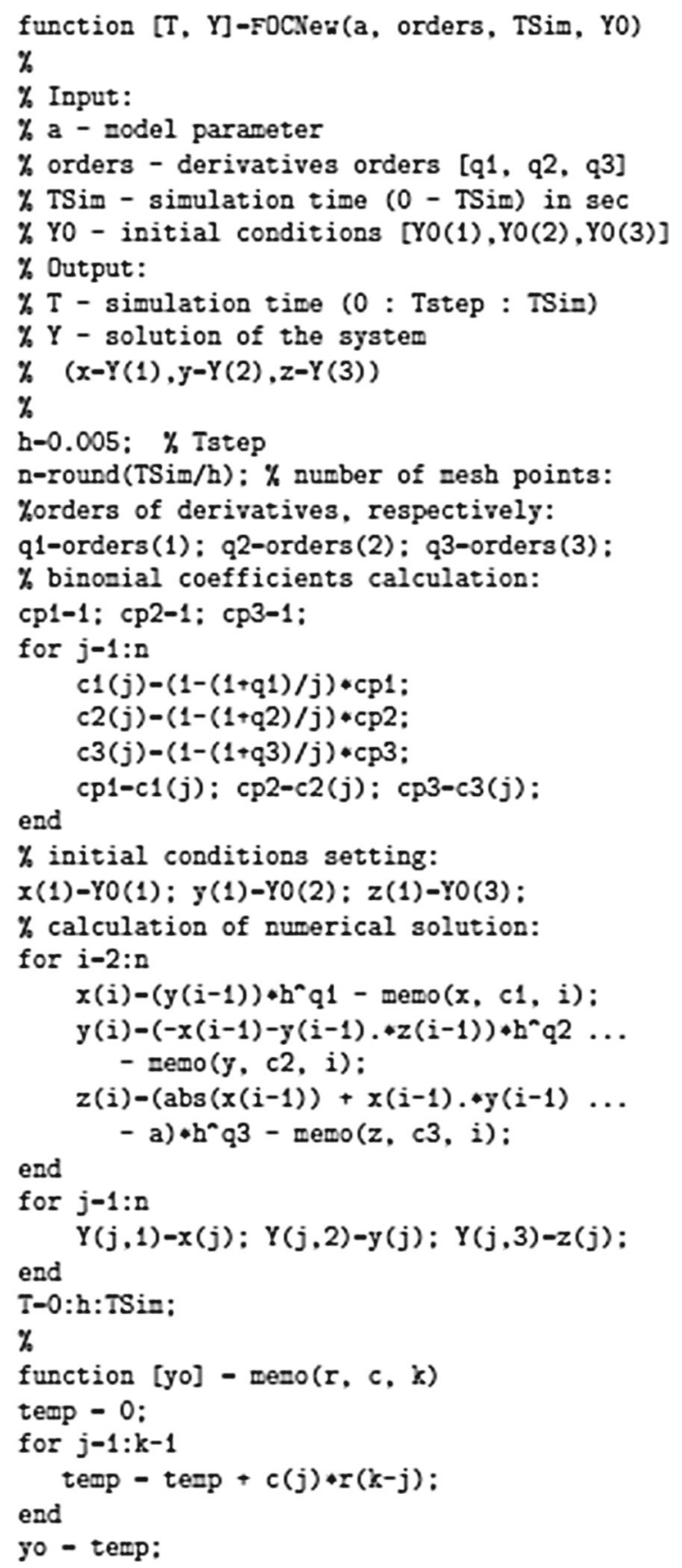




\section{References}

1. Cafagna, D., Grassi, G.: Elegant chaos in fractional-order system without equilibria. Math. Probl. Eng. 2013, 7 (2013). Article ID 380436

2. Cafagna, D., Grassi, G.: Chaos in a new fractional-order system without equilibrium points. Commun. Nonlinear Sci. Numer. Simul. 19(9), 2919-2927 (2014)

3. Cafagna, D., Grassi, G.: Fractional-order systems without equilibria: the first example of hyperchaos and its application to synchronization. Chin. Phys. B 24, 080502 (2015)

4. Caponetto, R., Dongola, G., Fortuna, L., Petráš, I.: Fractional Order Systems: Modeling and Control Applications. World Scientific, Singapore (2010)

5. Dudkowski, D., Jafari, S., Kapitaniak, T., Kuznetsov, N.V., Leonov, G.A., Prasad, A.: Hidden attractors in dynamical systems. Phys. Rep. 637, 1-50 (2016)

6. Jafari, S., Sprott, J.C., Nazarimehr, F.: Recent new examples of hidden attractors. Eur. Phys. J. Spec. Top. 224, 1469-1476 (2015)

7. Kingni, S.T., Pham, V.T., Jafari, S., Kol, G.R., Woafo, P.: Three-dimensional chaotic autonomous system with a circular equilibrium: analysis, circuit implementation and its fractional-order form. Circuits Syst. Signal Process. 35, 1933-1948 (2016)
8. Oldham, K.B., Spanier, J.: The Fractional Calculus. Academic Press, New York (1974)

9. Pham, V.T., Volos, C., Jafari, S., Wei, Z., Wang, X.: Constructing a novel no-equilibrium chaotic system. Int. J. Bifurcat. Chaos 24(5), 1450073 (2014)

10. Pham, V.T., Vaidyanathan, S., Volos, C., Jafari, S.: Hidden attractors in a chaotic system with an exponential nonlinear term. Eur. Phys. J. Spec. Top. 224, 1507-1517 (2015)

11. Pham, V.T., Volos, C., Jafari, S., Kapitaniak, T.: Coexistence of hidden chaotic attractors in a novel no-equilibrium system. Nonlinear Dyn. 87, 2001-2010 (2017)

12. Petráš, I.: Fractional-Order Nonlinear Systems. Springer, Berlin (2011)

13. Podlubny, I.: Fractional Differential Equations. Academic Press, San Diego (1999)

14. Westerlund, S., Ekstam, L.: Capacitor theory. IEEE Trans. Dielectr. Electr. Insul. 1(5), 826-839 (1994)

15. Wolf, A., Swift, J.B., Swinney, H.L., Vasano, J.A.: Determining Lyapunov exponents from a time series. Physica D 16(3), 285-317 (1985) 